\title{
EDITORIAL
}

\section{A birthday gift for GAVI}

\author{
In the year in which the GAVI Alliance celebrates its tenth anniversary, Bill and Melinda Gates \\ have pledged US $\$ 10$ billion in new funding. Judging from past successes, this is great news \\ for millions of children.
}

The GAVI Alliance was established in 2000 in response to the need to dramatically increase vaccine coverage for children in developing countries. Initially called the Global Alliance for Vaccination and Immunization, it is a coalition of partners, including national governments, international organizations, bilateral donors (donors that deal directly with one government), the vaccine production industry and non-governmental organizations. The alliance started with a $\$ 750$ million grant from the Bill and Melinda Gates Foundation, and Gro Harlem Brundtland, then Director General of the WHO and the first Chair of the GAVI Alliance board, pronounced its ambitious goal to be "protecting every child against killer diseases".

The importance of vaccines in combating infectious diseases is undeniable. The eradication of smallpox and near eradication of polio have only been possible because vaccines were available to prevent disease and break the chain of transmission. Many diseases that were threats one or two generations ago are now an afterthought in areas with active immunization programmes. However, although vaccination is now widely regarded as one of the most cost-effective health care interventions, when the GAVI Alliance was founded vaccination rates had started to decrease: the number of children immunized against common childhood diseases in developing nations rose from $5 \%$ to $80 \%$ between 1970 and 1990, but by 1999 it had decreased to $75 \%$. In addition, few new vaccines were being investigated.

During the first 10 years of the GAVI Alliance, more than 250 million children were vaccinated against various childhood diseases. The projected coverage with the diphtheria vaccine is up to nearly $80 \%$, coverage for hepatitis B is over $75 \%$ and coverage for Haemophilus influenzae type $\mathrm{b}(\mathrm{Hib})$ is also rapidly increasing. It has been estimated that by the end of this year, nearly 5 million deaths will have been averted owing to the efforts of the alliance. The recent introduction of a rotavirus vaccine is likely to add to this total; an estimated 500,000 children die of this infection annually.

To sustain and build on this success, the Bill and Melinda Gates Foundation has pledged \$10 billion to GAVI over the next 10 years, and it is estimated that this will allow up to 8 million lives to be saved. Over the next 5 years, GAVI hopes to make the pneumococcal and rotavirus vaccines available in 42 and 44 countries, respectively. Other vaccines that should be available in this time frame will target typhoid, Japanese encephalitis, rubella and cervical cancer caused by human papillomavirus. Furthermore, coverage of routine childhood diseases, including measles, diphtheria and whooping cough, should be increased to $90 \%$.

Part of GAVI's success lies in its effects on the economics of vaccine development and production. With its ability to provide a large guaranteed market, GAVI has made the development of vaccines for use in developing nations more attractive to pharmaceutical companies, which has increased competition and, as a result, lowered prices. For example, the number of companies that produce the pentavalent vaccine, which protects against Hib, diphtheria, pertussis, tetanus and hepatitis $\mathrm{B}$, has increased from one to four, and the price has recently decreased by $22 \%$ to below $\$ 3$ per dose. Through the efforts of GAVI, vaccines are also reaching developing countries more rapidly than in the past. Whereas it used to take 15-20 years to bring a vaccine to these countries, the pentavalent vaccine was being used in several developing nations within a decade of its introduction in developed countries. GAVI is also planning to bring the human papillomavirus vaccine, which has only been available in the United States since 2006, to developing nations. As UNICEF Deputy Executive Director Saad Houry remarked: "Clearly, industry understands and responds to a market, regardless of whether that market is in poor or rich countries. The Alliance's model is beginning to work, and we are optimistic that the trend will continue, as competition and demand increase over time."

The increased funding for GAVI is welcome news. But the funding should not be driven entirely by the Bill and Melinda Gates Foundation, and Bill Gates himself has encouraged others to step up and invest. National governments cannot let their attention and willingness to fund this programme, and others like it, slacken, even during economic downturns and when the focus on other international problems, such as global warming, increases. If we truly succeed in making this the 'decade of vaccines', great and sustainable improvements to the health of children in developing nations can be expected. 